\title{
NEW PLASTIC OPERATION FOR CORRECTING THE REFRACTIVE ERROR OF APHAKIC EYES BY CHANGING THE CORNEAL CURVATURE* \\ PRELIMINARY REPORT
}

BY

TADEUSZ KRWAWICZ

Lublin, Poland

THE problem of correcting the refractive error of an aphakic eye other than by prescribing the cumbersome cataract lenses still remains open, in spite of the excellent work done in this field by Ridley, and all its modifications. In search of a method which might combine greater safety for the patient with a simpler operative technique, an operation has been devised which consists in implanting a small plastic lens or a lamellar corneal graft between the lamellae of the central part of the cornea to increase the refractive power of the eye.

From the first stage of this enterprise, which comprised 52 experiments on rabbit eyes (Krwawicz, 1960), the following conclusions have been drawn:

(1) Intracorneal implantation of a plastic lens changes the refraction of the eye.

(2) After the implantation of the plastic lens the cornea heals without signs of irritation or reduction in clarity, but there is danger of a late reaction on the part of the corneal tissue, which may cause opacification.

(3) The refractive effect of the implanted lens continues even if the lens is removed after some days. This can be explained by the fact that when the lens is introduced between the two layers of the split cornea, the anterior layer is displaced towards the centre of the cornea by the thickness of the lens. This dislocation is permanently fixed by the healing process, so that the cornea cannot regain its previous shape, even if the lens is removed. The gap between the two layers of the cornea which remains after the removal of the lens then fills with clear corneal tissue, and only a delicate, annular scar marks the place where the edge of the lens had rested.

(4) Intracorneal implantation of a lamellar corneal graft distinctly changes the corneal curvature.

The results of these experiments encouraged a trial of the method on human eyes, first on those to be enucleated because of a neoplastic process. In these cases, too, the immediate effect was positive, viz. there was a distinct forward bulging of the part of the cornea overlying the implanted lens, the healing was free from complications, and the clarity of the cornea remained unimpaired.

Being now assured that the temporary presence of the plastic lens inside the corneal stroma had no harmful effect, and at the same time bearing in mind

* Received for publication January 1, 1960. 
the observation that the change in the corneal refraction persisted after its removal, the method was applied to correct refractive errors in eight aphakic eyes in seven patients.

\section{Operative Technique}

Under local anaesthesia, fixing sutures are placed in the lids and in the superior and inferior recti. Near the corneal margin a vertical incision, $4 \mathrm{~mm}$. long and deep enough to penetrate into the deeper layers of the stroma, is made with a small keratome (Fig. 1). From this incision the corneal stroma is split with a flat knife, and a pocket is formed in the central part of the cornea, corresponding in shape and size to the lens to be implanted (Fig. 2).

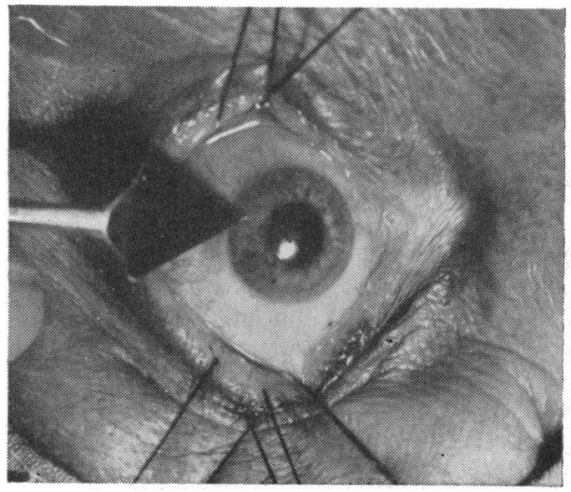

Fig. 1.-An incision about $4 \mathrm{~mm}$. long and penetrating into the deeper layers of the stroma is made at the limbus.

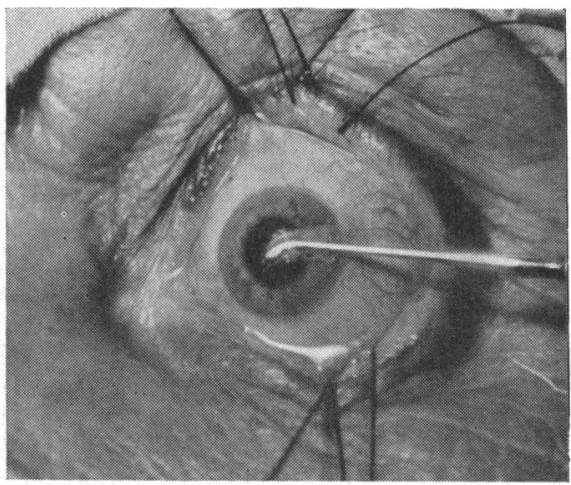

FIG. 2.-The corneal pocket is formed with a small flat knife.

The edge of the incision is then lifted with a spatula, and the plastic lens is introduced with a thin spatula into the incision, pushed through the channel between the split layers of the cornea, and finally placed at the bottom of the pocket, i.e. in the centre of the cornea (Figs 3 and 4, and Fig. 5, opposite).

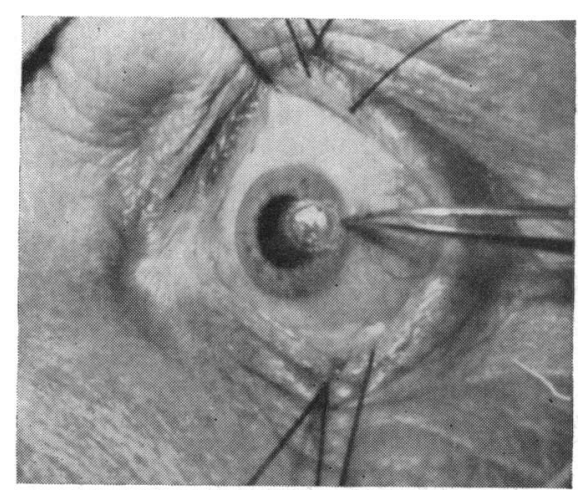

FIG. 3.-The plastic lens is introduced into the pocket by means of a forceps.

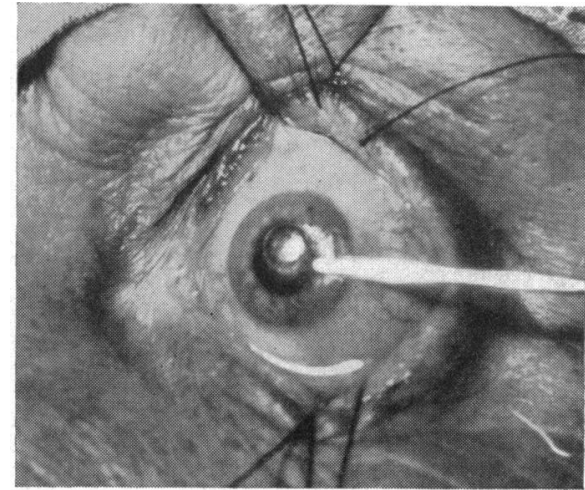

FIG. 4.-The lens is pushed with a spatula towards the centre of the cornea. 
Atropine, antibiotics, and later cortisone are administered locally. The patient is allowed to move freely, and the monocular dressing is removed on the following day.

After 8 to 10 days, when the wound produced by the procedure described above has healed, the plastic lens is removed by pulling it out with a forceps through an incision in the cornea. This is made on the same side as the original incision, but close to the edge of the lens (Figs 6, 7, and 8).

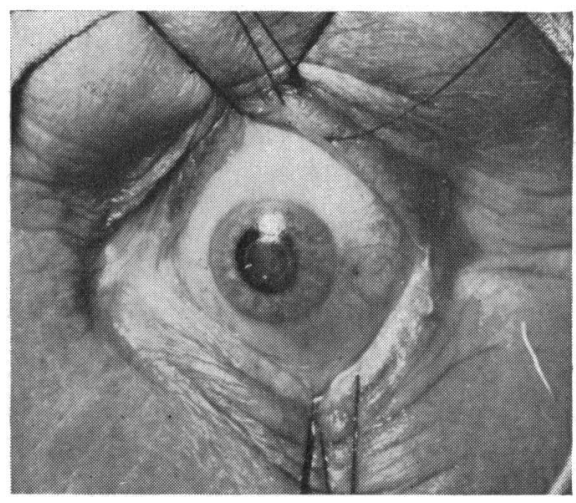

Fig. 5.-Plastic lens in place.

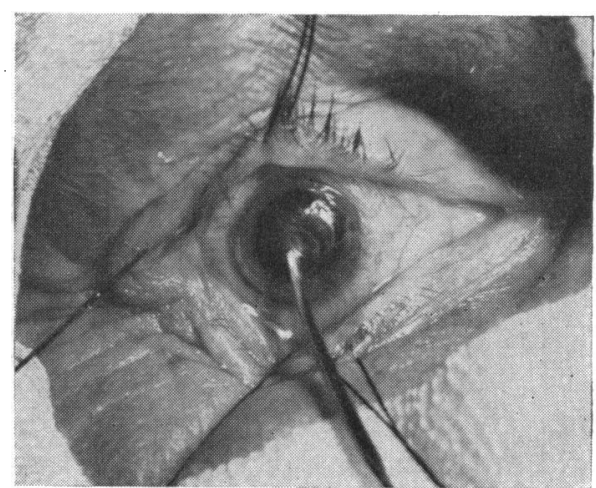

Fig. 7.-Pressure is applied to displace the lens. edge of the implanted lens.

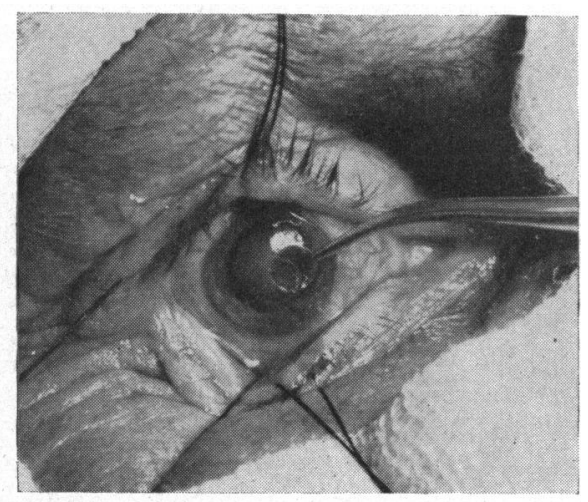

Fig. 8. The lens is removed with forceps.

Again atropine, antibiotics, and after some days corticosteroids are put into the conjunctival sac, and a uniocular dressing is applied for 24 to 48 hours. 
Results

This operation was performed on eight eyes (Table), all of which were emmetropic.

TABLE

RESULTS IN EIGHT EYES

\begin{tabular}{c|c|c|c|c|c|c}
\hline Case No. & Sex & $\begin{array}{c}\text { Age } \\
\text { (yrs) }\end{array}$ & Eye & $\begin{array}{c}\text { Follow-up } \\
\text { (mths) }\end{array}$ & $\begin{array}{c}\text { Post-Operative } \\
\text { Visual Acuity } \\
\text { (uncorrected) }\end{array}$ & Remarks \\
\hline 1 & F & 60 & Right & 7 & $5 / 15$ & \\
\hline 2 & F & 42 & Right & 6 & $5 / 15$ & $\begin{array}{c}\text { Infiltration, plastic lens } \\
\text { removed after 48 hrs }\end{array}$ \\
\hline 3 & & & Left & 4 & $5 / 15$ & \\
\hline 4 & F & 48 & Left & 4 & $5 / 20$ & \\
\hline 5 & F & 50 & Left & 3 & $5 / 15$ & \\
\hline 6 & F & 46 & Right & 3 & $5 / 30$ & \\
\hline 7 & M & 37 & Left & 3 & $5 / 30$ & Traumatic cataract \\
\hline 8 & F & 48 & Right & 2 & $5 / 30$ & \\
\hline
\end{tabular}

In six (Cases 1, 3, 4, 5, 6, and 8), the intracorneal implantation of the plastic lens came first, and then, 10 to 14 days after the removal of the implant, the cataract was extracted. Only in two instances (Cases 2 and 7) did the extraction of the cataract precede the plastic operation, the former sequence being preferred for technical reasons; Figs 9 and 10 show the final results obtained in these eyes.

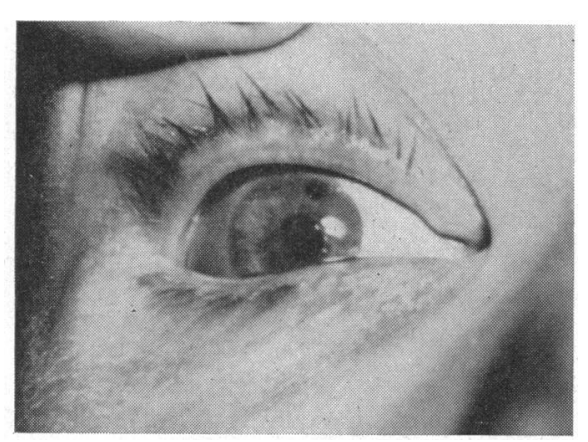

Fig. 9.-Final result, Case 2.

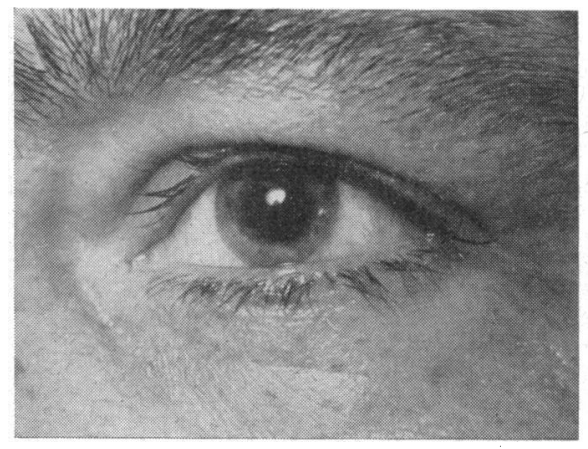

FIG. 10.-Final result, Case 7.

In almost all cases the healing process following the implantation of the lens progressed smoothly. There were no signs of a major irritation of the eye, and the split cornea, both in front of the lens and behind it, remained clear. 
In one patient only (Case 2) a small infiltration appeared in the lower angle of the pocket 48 hours after the operation. The artificial lens was immediately removed, the infiltration disappeared, and the cornea remained perfectly clear. In this case the visual result was 5/15 (Fig. 9).

The course of healing after the removal of the implanted lens closely resembled that following lamellar keratoplasty and was free from complications.

The visual results*, which were in four cases $5 / 15$, in one $5 / 20$, and in three $5 / 30$, can be regarded as encouraging if they are compared with those which can normally be expected from an uncorrected aphakic eye. The observation period ( 2 to 7 months) may, of course, be too short to exclude the possibility of a change in the optical effect of the operation, though no such tendency has been noticed so far.

A somewhat different approach, consisting in a permanent intracorneal implantation of a corneal graft, has been carried out in only one patient so far. After 2 months the graft remains clear, and the uncorrected visual acuity is $5 / 30$. Of course, there is no assurance that the graft will not be partly or completely absorbed later on. Should the effect prove permanent, further research in this direction would be indicated.

\section{Conclusions}

The clinical results of the operation described above demonstrate that it is possible to implant a temporary intracorneal plastic lens to change the curvature of the cornea, and consequently to correct the refractive error of an aphakic eye. The operative risk is small and, even if the visual acuity is not improved, little or no harm is done to the eye.

The correction of the refractive error which we were able to obtain was only partial, though in some cases quite remarkable. The problem demands further investigation, and above all it is necessary to develop methods which will assure a more precise and adequate measurement of the correction.

\section{Summary}

The first results are reported of the clinical application of a new corneal operation aimed at correcting the refractive error of aphakic eyes by changing the curvature of the central part of the cornea. The procedure consists in the intralamellar implantation of a small plastic lens, which is removed when the change in the corneal curvature has been fixed by the healing process.

The results so far obtained by this method can be regarded as promising, the more so in that the operative risk is small. This being only a preliminary report, the need is stressed for further testing in clinical practice.

KRWAWICZ, T. (1960). Klin. oczna, 30, 229.

\section{REFERENCE}

* Only the axial visual acuity is reported here. The result of a more detailed examination of the refraction will be dealt with at a later date. 